\title{
Sensitivity to material contrast in homogenization of random particle composites as micropolar continua
}

\author{
Emanuele Reccia ${ }^{1 *}$, Maria Laura De Bellis ${ }^{2}$, Patrizia Trovalusci ${ }^{1}$, \\ Renato Masiani ${ }^{1}$ \\ ${ }^{1}$ Department of Structural and Geotechnical Engineering, Sapienza University of Rome, \\ ${ }^{2}$ Department of Innovation Engineering, University of Salento, Lecce, Italy \\ *corresponding author emanuele.reccia@uniroma1.it
}

\begin{abstract}
Several composite materials used in engineering - such as ceramic/ metal matrix composites, concrete, masonry-like/geo-materials and innovative meta-materials - have internal micro-structures characterized by a random distribution of inclusions (particles) embedded in a matrix. Their structural response is highly influenced not only by the mechanical properties of components, but also by the shape, size and position of the inclusions.

In this work, we adopt a statistically-based micropolar homogenization procedure, to obtain the overall elastic properties of homogeneous micropolar continua able to naturally account for scale and skew-symmetric shear effects. Attention is paid to the sensitivity to material contrast, defined as the mismatch between classical and micropolar constitutive properties of matrix and inclusions. A statistical specifically conceived convergence criterion is adopted which allow us to identify the REV (Representative Volume Element) for any value of material contrast.
\end{abstract}

Keywords - Particle Composites, Micropolar continua, ScaleDependent Statistical Homogenization, Representative Volume Element

\section{Introduction}

Particle composites are a special class of heterogeneous materials exhibiting an internal microstructure characterized by particles randomly distributed 
within a continuous matrix phase. Composites showing such a topology are widespread in many engineering fields, from aerospace to restoration of architectural heritage. Besides typical examples of short-fiber reinforced materials, ceramic- and metal-matrix composites, poly-crystals, concrete and porous rocks, also innovative smart materials and meta-materials can be counted among particle composites. The study of these materials certainly requires a through knowledge of the micromechanical elastic response and an appropriate estimation of their overall structural behavior. This stage is a prerequisite for the design of new smart materials with properly tuned properties and it can guide in the choice of constituent materials and in the definition of percentage and shape of the inclusions to be embedded in the matrix, in a perspective of structural optimization.

The discontinuous and heterogeneous nature of these materials has a strong relevance at the microscale [30]. Based on this observation, Discrete Element Models [10, 9], successfully adopted to study masonry materials [22, 3], have been also applied to particulate composites [18, 42], and fibre reinforced composites [20]. The main drawback of this kind of approaches is related to the considerably high computational costs required, so that analyses may be limited to small portions of structure.

A possible way out is the use of continuous models within the framework of multiscale procedures. Multiscale approaches have been used by many authors for the purpose of obtaining equivalent continua able to take into account, at a coarse scale, the internal microstructure emerging from embedded finer scales [14, 29, 12, 5, 32. Homogenization techniques have been exploited to study several typologies of materials, in particular to model those having periodic microstructures via equivalent homogeneous continua both in two and three dimensional frameworks. Within this approaches several authors proposed to properly take into account internal material length scales, resorting to non classical continuum modelling (higher order, micropolar and other kind of multifield continua) [35, 15, 24, 7, 37, 31, 8, 23, 33, 41, 11, 1, 2, 4, 17].

Among various continua, the micropolar continuum proves to be very effective in representing the scale dependency and skew-symmetric material response of orthotropic periodic media [27, 40].

Homogenization techniques have been also adopted to model non-periodic media. In the case of materials exhibiting a random microstructure, such as random particle composites, the classical homogenization techniques adopted for periodic media are not suitable. This because the concept of the Representative Volume Element (RVE) (a volume portion of the heterogeneous material that is statistically representative of the microstructural features of the composite [21]), well-established for periodic media, calls for the need of very large (theoretically infinite) material domains and depending on the 
macroscopic body size, this may invalidate the scale separation commonly assumed in continuum mechanics [25, 26]. The RVE cannot be detected $a$ priori, but it is an unknown of the homogenization problem.

In the last few decades, procedures based on the solution of Boundary Value Problems (BVPs) on finite-size mesoscales have been developed in order to apply homogenization techniques to materials with random microstructure [34, 28, 16, 43.

According to the statistically-based approach proposed in [25], the evaluation of the overall elastic response of random composites and the identification of the RVE size can be performed by exploiting the convergence between two hierarchies of bounds, that are the solutions of BVPs defined on Statistical Volume Elements (SVEs) undergoing fully displacement (Dirichlet) and traction (Neumann) boundary conditions; providing upper and lower bounds of the solution, respectively. This approach has been extended to the case of micropolar continua at both microscopic and macroscopic scale in [39] and specialized to the study of particle composites in [38, 36].

In this paper, we apply this statistically-based homogenization procedure to study particle composites characterized by different mismatch between the classical and micropolar elastic properties of matrix and inclusions. The numerical approach aims at detecting the appropriate RVE size defined for random particle composites modeled as micropolar continua.

We focus on the study of particle composite materials in which typically the volume fraction of inclusions $\rho=V_{i} /\left(V_{i}+V_{m}\right)$ (with $V_{i}$ and $V_{m}$ being the volumes of inclusions and matrix, respectively) is lower than $40 \%$. Under this assumption, the material can be described at the microscopic scale as an aggregate of randomly distributed inclusions that are embedded into a base matrix, either softer or stiffer. At the macroscale the heterogeneous material is replaced by an equivalent homogeneous material.

The micropolar continuum is adopted at both the macroscale and the microscale to properly account for scale effects related to the fact that in such materials the typical size of inclusions $d$ is not negligible with respect to the structural characteristic length $L$. In this case the classical homogenization fails since the scale separation principle is not valid any more.

The satisfaction of an energetically-based generalized macro-homogeneity condition provides the boundary conditions to be adopted in the BVPs defined on each statistical realization of the microstructure (SVE). The estimation of equivalent elastic, classical and micropolar, moduli is repeated for several realizations of the random heterogeneous medium. This corresponds to ideally move a window within the actual material domain. The window size is then increased until a properly established convergence criterion is satisfied. The adopted criterion holds true for any value of the material con- 
trast (i.e. the ratio between the elastic moduli of matrix and inclusions). Once the convergence is achieved, the RVE size is detected and the related homogenized moduli are estimated.

The results of the statistically based micropolar homogenization procedure strongly depend on the material properties of the constituents. For this reason, parametric analyses, performed to investigate the converge trends as the mismatch in the elastic properties of matrix and inclusions vary, are useful to better characterize these kind of materials.

The outline of the paper is as follows. In Section 2 the micropolar homogenization approach is introduced. The governing equations at both the macroscopic and the microscopic scales are recalled and a generalized macrohomogeneity condition establishes the energy equivalence between them. In Section 3 the statistically-based procedure adopted to take into account the randomness in the material parameters distribution is briefly described. Numerical results are presented and discussed in Section 4 where parametric numerical analyses are carried out in order to investigate the influence of the mismatch of material parameters at the microscales on the overall elastic behaviour. Finally, in Section 5 some concluding remarks are presented.

\section{Micropolar homogenization}

At both macroscopic and microscopic scales, micropolar continua are taken into account. The governing equations are formally the same except for the constitutive law that is not 'a priori' defined at the macroscopic level, but directly descends from the lower level as result of the homogenization procedure. In the following, lower case letters always refer to micro while upper case letters to macro scale. Considering a two-dimensional (2D) case, each material point is characterized by two displacement and one rotation components $\left\{u_{1}, u_{2}, \phi_{3}\right\}$ and $\left\{U_{1}, U_{2}, \Phi_{3}\right\}$ at micro- and macro-scale, respectively.

Within the framework of a linearized theory, the kinematics of the micropolar continua are governed by the compatibility equations in which the symmetric and skew-symmetric parts of the strain are explicitly derived $(i, j=1,2)$ :

$$
\begin{aligned}
\varepsilon_{i j} & =\frac{1}{2}\left(u_{i, j}+u_{j, i}\right), \\
\alpha_{12} & =\frac{1}{2}\left(u_{1,2}-u_{1,2}\right)-e_{312} \phi_{3} \\
\kappa_{3 i} & =\phi_{3, i}
\end{aligned}
$$

where $\left(\varepsilon_{i j}\right)$ is the symmetric strain tensor, $\left(\alpha_{12}\right)$ the skew-symmetric strain 
and $\left(\kappa_{i 3}\right)$ the curvature tensor at the microscopic level and

$$
\begin{aligned}
E_{i j} & =\frac{1}{2}\left(U_{i, j}+U_{j, i}\right), \\
A_{12} & =\frac{1}{2}\left(U_{1,2}-U_{2,1}\right)-e_{312} \phi_{3}, \\
K_{3 i} & =\Phi_{3, i},
\end{aligned}
$$

where $\left(E_{i j}\right)$ is the symmetric strain tensor, $\left(A_{12}\right)$ the skew-symmetric strain and $K_{3 i}$ the curvature tensor at the microscopic level.

The stress tensors work conjugated to the strains respectively are $\left(\sigma_{i j}\right)$, $\left(\beta_{12}\right)$ and $\left(\mu_{3 i}\right)$ at the microscopic level and $\left(\Sigma_{i j}\right),\left(B_{12}\right)$ and $\left(M_{3 i}\right)$ at the macroscopic level.

We assume that at the lower level each material phase is characterized by linear elastic isotropic micropolar behavior with the stress-strain relations written as:

$$
\left[\begin{array}{c}
\sigma_{11} \\
\sigma_{22} \\
\sigma_{12} \\
\beta_{12} \\
\mu_{31} \\
\mu_{32}
\end{array}\right]=\left[\begin{array}{cccccc}
\lambda+2 \mu & \lambda & 0 & 0 & 0 & 0 \\
\lambda & \lambda+2 \mu & 0 & 0 & 0 & 0 \\
0 & 0 & 2 \mu & 0 & 0 & 0 \\
0 & 0 & 0 & 2 \mu_{c} & 0 & 0 \\
0 & 0 & 0 & 0 & 2 \mu l_{c}^{2} & 0 \\
0 & 0 & 0 & 0 & 0 & 2 \mu l_{c}^{2}
\end{array}\right]\left[\begin{array}{c}
\varepsilon_{11} \\
\varepsilon_{22} \\
\varepsilon_{12} \\
\alpha_{12} \\
\kappa_{31} \\
\kappa_{32}
\end{array}\right]
$$

which involves four independent elastic constitutive parameters: the Lamé constants $\lambda$ and $\mu, \mu_{c}$ the micropolar shear modulus and the so-called characteristic length $l_{c}$, which is responsible for the rotational stiffness (See 38], [13] for a more detailed description of the material constants adopted).

At the macroscopic level, the general anisotropic 2D stress-strain relations, write:

$$
\left[\begin{array}{c}
\Sigma_{11} \\
\Sigma_{22} \\
\Sigma_{12} \\
B_{12} \\
M_{31} \\
M_{32}
\end{array}\right]=\left[\begin{array}{llllll}
\mathbb{A}_{1111} & \mathbb{A}_{1122} & \mathbb{A}_{1112} & \mathbb{D}_{1112} & \mathbb{F}_{1131} & \mathbb{F}_{1132} \\
\mathbb{A}_{2211} & \mathbb{A}_{2222} & \mathbb{A}_{2212} & \mathbb{D}_{2212} & \mathbb{F}_{2231} & \mathbb{F}_{2232} \\
\mathbb{A}_{1211} & \mathbb{A}_{1222} & \mathbb{A}_{1212} & \mathbb{D}_{1212} & \mathbb{F}_{1231} & \mathbb{F}_{1232} \\
\mathbb{D}_{1211} & \mathbb{D}_{1222} & \mathbb{D}_{1212} & \mathbb{B}_{1212} & \mathbb{G}_{1231} & \mathbb{G}_{1232} \\
\mathbb{F}_{3111} & \mathbb{F}_{3122} & \mathbb{F}_{3112} & \mathbb{G}_{3112} & \mathbb{C}_{3131} & \mathbb{C}_{3132} \\
\mathbb{F}_{3211} & \mathbb{F}_{3222} & \mathbb{F}_{3212} & \mathbb{G}_{3212} & \mathbb{C}_{3231} & \mathbb{C}_{3232}
\end{array}\right]\left[\begin{array}{c}
E_{11} \\
E_{22} \\
E_{12} \\
A_{12} \\
K_{31} \\
K_{32}
\end{array}\right] .
$$

At this level, the equivalent moduli are obtained via the homogenization procedure proposed in [39], based on a generalization on the original macrohomogeneity condition, proposed by Hill in [19]:

$$
\Sigma_{i j} E_{i j}+B_{12} A_{12}+M_{3 i} K_{3 i}=\frac{1}{A_{\delta}} \int_{\mathcal{B}_{\delta}}\left(\sigma_{i j} \varepsilon_{i j}+\beta_{12} \alpha_{12}+\mu_{3 i} \kappa_{3 i}\right) d A
$$


where the usual summation convention for repeated indices is used through and the symmetric and skew-symmetric strain and stress components are splitted, consistently with the above definitions, in order to separately investigate their effects. We can notice that, in the case of centrosymmetric (i.e. non-chiral) materials, the components of the tensors $\mathbb{D}, \mathbb{F}$ and $\mathbb{G}$ vanish.

Equation (5) states the equivalence between the stress power evaluated at the microscopic level within a window $\mathcal{B}_{\delta}$, occupying the region of area $A_{\delta}$, and the stress power at a macroscopic point. Directly from the fulfilment of Equation (5) a set of Dirichlet and Neumann boundary conditions on the boundary $\partial \mathcal{B}_{\delta}$ derive, applied at the micro-scale for the solution of the BVP:

$$
u_{i}=E_{i j} x_{j}, \quad \varphi_{3}=\frac{1}{2} e_{k j 3} A_{k j}+K_{3 j} x_{j} \quad \text { on } \partial \mathcal{B}_{\delta},
$$

with $x_{j}$ being the $j$-th coordinate of the generic point on the boundary, with respect to a reference system with origin in the geometric center of the RVE, and the Neumann boundary conditions are:

$$
t_{i}=\left(\Sigma_{i j}+B_{i j}\right) n_{j}, \quad m_{3}=m_{3}^{o}+M_{3 i} n_{i} \quad \text { on } \partial \mathcal{B}_{\delta},
$$

where $t_{i}$ and $m_{3}$ are tractions and surface couples, $m_{3}^{o}=-\int_{\partial \mathcal{B}_{\delta}} e_{3 j l} x_{j} B_{k l} n_{k}$ being the moment imposed to ensure the moment balance in the presence of skew-symmetric shear.

\section{Statistically-based homogenization proce- dure}

The purpose of the statistically-based homogenization procedure is the evaluation of the elastic scale-dependent effective response of random particle composites, basing on the detection of the RVE size, $L_{R V E}$. Due to the lack of periodicity in the microstructure, it is, indeed, impossible to 'a priori' identify, via topological observations, a representative portion of the heterogeneous material, thus $L_{R V E}$ becomes an additional unknown of the problem with respect to periodic media [26].

In the following we recall the main steps of the procedure, while for a detailed description refer to the articles [39, 38].

1. The heterogeneous medium is modeled as a two-phase material with circular inclusions, of diameter $d$, randomly distributed within a base matrix. The assumptions of statistical homogeneity and mean-ergodicity hold. 
2. The nominal volume fraction is kept fixed, equal to $\rho=40 \%$, while both number and position of inclusions can randomly vary.

3. For a fixed value of the scale parameter, $\delta=L / d$ (being $L$ the side of a square test window) a number of realization of the microstructure $\mathcal{B}_{\delta}(\omega)$, where $\omega$ is an elementary event over a sample space, is generated. The number and position of inclusions varies within the window, adopting a Poisson's distribution (SVEs, i.e. finite domains involving statistical scattering), satisfying a hard-core condition which prevents the overlapping between disks. This corresponds to a moving windows technique.

4. For each realization $\mathcal{B}_{\delta}(\omega)$, both Dirichlet and Neumann BVPs are solved, accounting for classical and micropolar deformation modes, and the relevant homogenized constitutive moduli are numerically determined.

5. When, after a number of simulations $N_{\delta}$, the average value of an elastic modulus falls within a confidence interval, set at $95 \%$ over a normal distribution $\frac{1,96 C V}{\sqrt{N_{\delta}}} \leq t o l$, the procedure stops. The coefficient of variation $\mathrm{CV}$ is defined depending on the material dispersion, $C V=$ $\sigma /\left\langle X_{\delta}\right\rangle$, with $\sigma$ being the standard variation and $\left\langle X_{\delta}\right\rangle$ the average of $X$ estimated at a given $\delta$ and $t o l$ is a given tolerance.

6. The moving window procedure is then repeated for increasing values of the scale factor, $\delta$, until $N_{\delta}$ satisfies the aforementioned condition. The most unfavorable case is considered: at this stage we assume that the RVE is identified.

7. The overall modulus is finally estimated as the average value between the Dirichlet and Neumann solutions evaluated at $\delta=\delta_{R E V}=L_{R E V} / d$.

\section{Parametric numerical analyses}

Two cases of particle-based composites are considered:

- Material "A", in which stiff inclusions are embedded in a soft matrix;

- Material "B", in which soft inclusions are embedded in a stiff matrix.

The constitutive behavior of the constituents, for both materials A and $\mathrm{B}$ is assumed linear elastic and isotropic, as described in Section 2, For the 
classical part, the material contrast has been defined as the ratio $E_{i} / E_{m}$, with $E_{i}$ and $E_{m}$ being the elastic moduli of inclusions and matrix, respectively. While for the micropolar part, the material contrast has been defined as the ratio $l_{c i} / l_{c m}$, with $l_{c i}$ and $l_{c m}$ being the characteristic lengths of inclusions and matrix, respectively. For both cases A and B, the simulations are performed by varying the values of material contrast as reported in Table 1. The limit cases of the ratios tending to $0(\mathrm{~A})$ or to $\infty$ (B) describe composites with rigid inclusions or pores, while the special case of ratios equal to 1 refers to homogeneous materials, [6].

\begin{tabular}{|c|c|c|c|}
\hline Material A & A1 & $\mathrm{A} 2$ & A3 \\
\hline & $\begin{array}{l}\frac{E_{i}}{E_{m}}=10 ; \\
\frac{l_{c i}}{l_{c m}}=10\end{array}$ & $\begin{array}{l}\frac{E_{i}}{E_{m}}=10^{2} \\
\frac{l_{c i}}{l_{c m}}=10^{2}\end{array}$ & $\begin{array}{l}\frac{E_{i}}{E_{m}}=10^{3} \\
\frac{l_{c i}}{l_{c m}}=10^{3}\end{array}$ \\
\hline Material B & B1 & $\mathrm{B} 2$ & B3 \\
\hline & $\begin{array}{l}\frac{E_{i}}{E_{m}}=10^{-1} \\
\frac{l_{c i}}{l_{c m}}=10^{-1}\end{array}$ & $\begin{array}{l}\frac{E_{i}}{E_{m}}=10^{-2} ; \\
\frac{l_{c i}}{l_{c m}}=10^{-2}\end{array}$ & $\begin{array}{l}\frac{E_{i}}{E_{m}}=10^{-3} \\
\frac{l_{c i}}{l_{c m}}=10^{-3}\end{array}$ \\
\hline
\end{tabular}

Table 1: Classical and micropolar material contrasts

As the homogenized behavior has been found to be isotropic, we select two representative material parameters: the bulk modulus $\bar{K}$, for the classical part, and the characteristic length, related to the bending and skew shear moduli, $\overline{l_{c}}$ for the micropolar part.

Referring to the $2 \mathrm{D}$ constitutive equations at the macroscale (4), the effective bulk modulus and the homogenized characteristic length are respectively defined as:

$$
\begin{aligned}
\bar{K} & =2 \overline{\mathbb{A}}_{1122}+\overline{\mathbb{A}}_{1212} \\
\overline{l_{c}} & =\sqrt{\left(\overline{\mathbb{C}}_{3131}+\overline{\mathbb{C}}_{3232}\right) / \overline{\mathbb{B}}_{1212}}
\end{aligned}
$$

The statistical procedure described in Section 3 is applied in order to perform the parametric analysis aimed at identifying the appropriate RVE 
size $\left(\delta_{R V E}\right)$ for materials $\mathrm{A}$ and $\mathrm{B}$ and the corresponding elastic, classical and micropolar moduli defined in Equation (8). Attention is paid to the convergence trend of these homogenized moduli.

In the Finite Element discretization, unstructured meshes of quadratic Lagrangian triangular elements are adopted. All the BVPs have been solved by using COMSOL Multiphysics ${ }^{\mathbb{B}}$ ) code.

The results of the parametric analyses are reported in Figure 1 and 2 , in which the average value of the homogenized bulk modulus $\langle\bar{K}>$ and the average value of the homogenized characteristic length $\left\langle\overline{l_{c}}\right\rangle$, obtained for the different material contrasts, are plotted versus the scale parameter $\delta=L / d$. Both $\left\langle\bar{K}>\right.$ and $\left\langle\bar{l}_{c}>\right.$ are normalized with respect to the values obtained at the RVE: $\left\langle\bar{K}_{R V E}>\right.$ and $\left\langle\bar{l}_{c R V E}>\right.$. In the figures, blue solid lines are referred to the solution of Neuman BVPs, while the red dashed lines to the solution of Dirichlet BVPs.

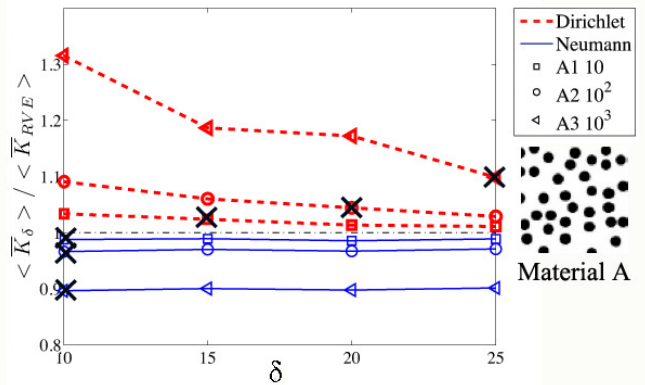

(a)

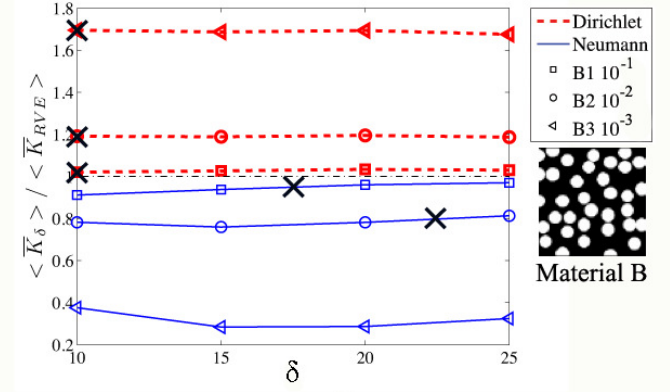

(b)

Figure 1: Material A/B: effective bulk moduli (normalized) $<\bar{K}_{\delta}>/<$ $\bar{K}_{R V E}>$ versus scale parameter $\delta$ for different contrasts. The "X" symbol indicate the value obtained at convergence

As regards to the bulk modulus $<\bar{K}>$, both the Dirichlet and the Neumann solutions converge to an average value falling within the confidence interval (step 5 of the procedure of Section 3). As indicated in Section 3 , step 6 , the value $\delta_{R V E}$ is then defined by taking into account the largest convergence value between Dirichlet and Neumann solutions. It is possible to observe that in the case of material A (Figure1 (a)), looking at the solution of the Neumann's BVPs, the convergence criterion is satisfied for values of $\delta$ lower than those obtained adopting Dirichlet BCs; while in the case of material B (Figure1 (b)) the opposite occurs.

In the latter case the gap between the two, Dirichlet and Neumann, solutions is bigger than in the former. This is due to the fact that material $\mathrm{B}$ needs more simulation to reach the convergence, as it will be explained in the following. 


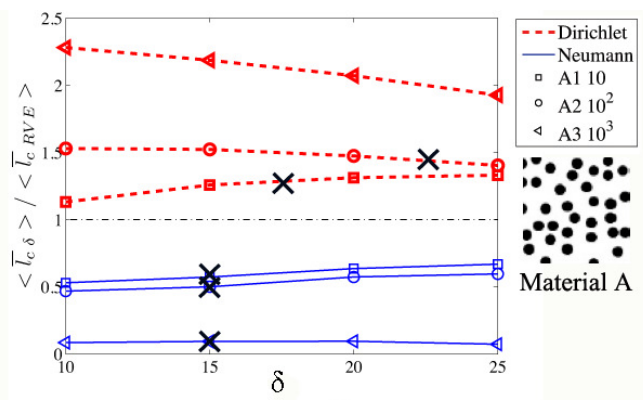

(a)

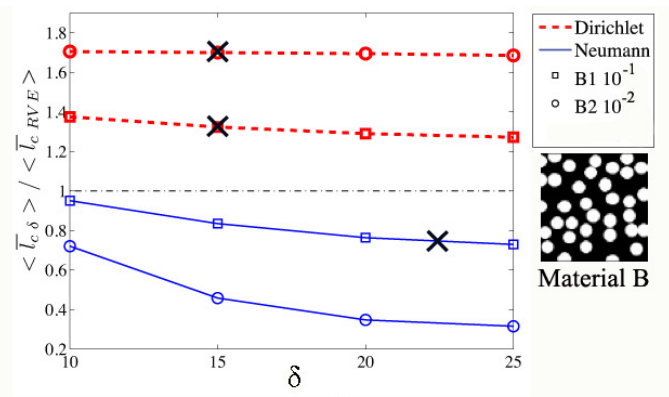

(b)

Figure 2: Material A/B: effective characteristic length (normalized) $\left\langle\bar{l}_{c \delta}>\right.$ $/<\bar{l}_{c R V E}>$ versus scale parameter $\delta$ for different contrasts. The "X" symbol indicate the value obtained at convergence

Looking at the characteristic length $\left\langle\overline{l_{c}}\right\rangle$, a similar trend may be observed (Figure 2). However, in this case the two bounds tend to become parallel as $\delta$ increases and the constant tangent indicates the convergence. The value of $\delta_{R V E}$ is then defined by taking into account the largest convergence value between Dirichlet and Neumann solutions.

Also in this case, material B calls for greater values of $\delta_{R E V}$, in particular for the solution of Neumann BVPs (Figure 2 (b)).

Concerning the convergence trend, for both the classical and micropolar material moduli, as $\delta$ increases, the results obtained by applying Dirichlet BCs and Neuman BCs tend to get closer. According to [26], when the values of classical and micropolar contrasts move away from 1 (homogeneous material), the distance between the two solutions increases as the contrast varies calling for the need of larger size windows for identifying the RVE size, $\delta_{R V E}$. The variation is more sensitive when the contrast decreases (material B).

Within the proposed statistical approach, the RVE can be reached independently on the material contrast basing on the criterion of the maximum number of simulations needed to obtain values that do not vary more than $5 \%$ as the window size increases.

Figures (1) and (2) illustrate this criterion, which always ensures the achievement of the RVE, also in those cases in which the value of the material contrast is very different from 1 .

More in detail, in Figures (3) and (4), the average moduli are plotted versus the number of simulations.

For the sake of brevity, among the several analysis performed for different material contrasts, only the results for materials A2 and B2 are reported, these being the intermediate contrasts of the two typologies of materials considered. Both the results obtained by applying Dirichlet and Neumann 
BCs are plotted.

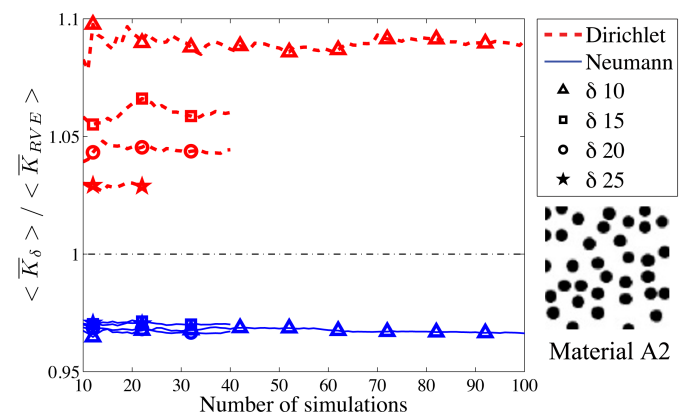

(a)

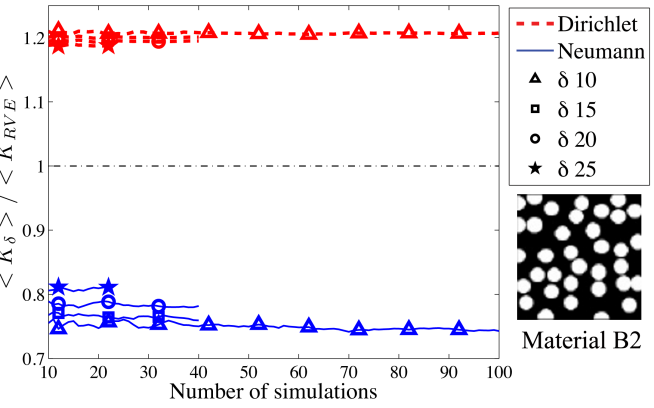

(b)

Figure 3: Material A2/B2: Average values of the effective bulk modulus $<\bar{K}_{\delta}>$ normalized to the convergence value $\left\langle\bar{K}_{R V E}>\right.$ versus the number of simulations performed for different window sizes.

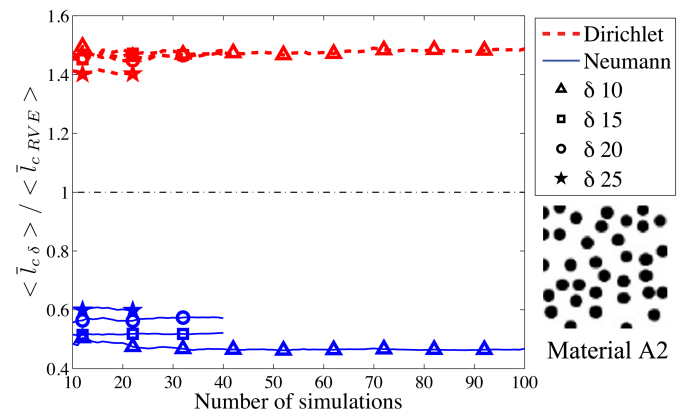

(a)

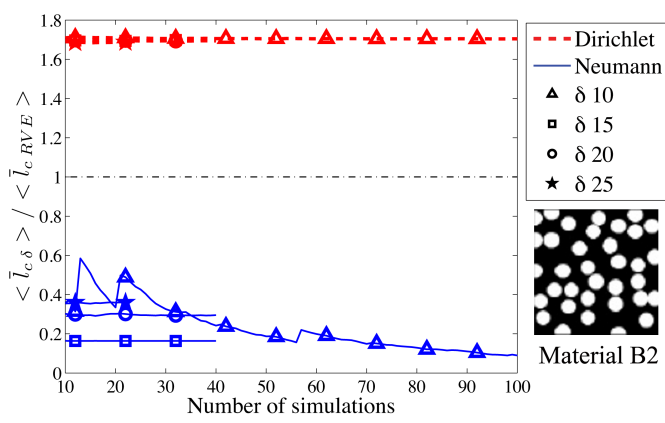

(b)

Figure 4: Material A2/B2: Average of values of the effctive characteristic length $\left\langle\bar{l}_{c \delta}>\right.$ normalized to the convergence value $<\bar{l}_{c R V E}>$ versus number of simulations performed for different window sizes.

In particular, Figure 3 shows the average values of the homogenized bulk modulus, $\left\langle\bar{K}_{\delta}>/<\bar{K}_{R V E}>\right.$ versus the number $N$ of simulations performed.

It is worth noting that the number of simulations $N_{\delta}$ needed for the convergence decreases as the scale factor $\delta$ increases. However, as noted before, a different trend characterizes the two materials: in the case of material A2 the Dirichlet BVPs solutions require a higher number of simulations with respect to the Neumann BVPs solutions, while the opposite occurs in the case of material B2. As defined above, the RVE size is obtained as the largest size between the convergence values of Dirichlet and Neumann BVPs solutions. In the case of the material A2 for instance, Neumann's solutions allow us to 
define $\delta_{R V E}=15$, while Dirichlet's solutions provide $\delta=20$. This latter value has been then considered as RVE value. The trend is opposite in the case of material B2.

Similar considerations may be drawn looking at Figure (4), in which the average value of the homogenized characteristic length $\left\langle\bar{l}_{c \delta}>/<\bar{l}_{c R V E}>\right.$ are plotted versus the number of simulations performed $N$. As previously noted, in the case of material $\mathrm{B}$, the number of simulation needed to reach the convergence is higher with respect to material $\mathrm{A}$.

The differences in terms of convergence trend of classical and micropolar moduli in the material cases $\mathrm{A}$ and $\mathrm{B}$ depend on the different dispersion of results are shown in Figures 5 and 6, reporting the Coefficient of Variation $C V$ (Section 3, step 5) versus $\delta$.
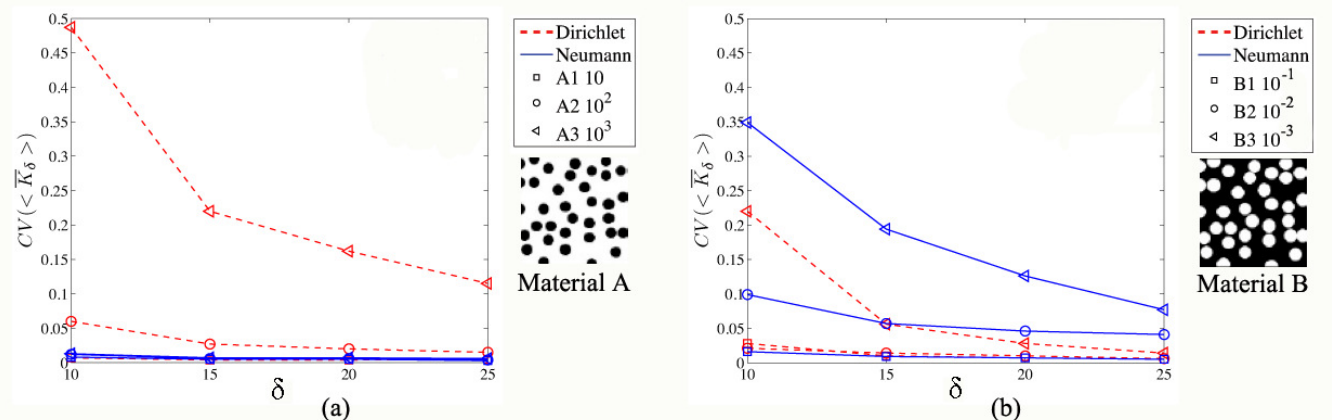

Figure 5: Material A/B: Coefficient of variation of the effective bulk modulus $<\bar{K}_{\delta}>$
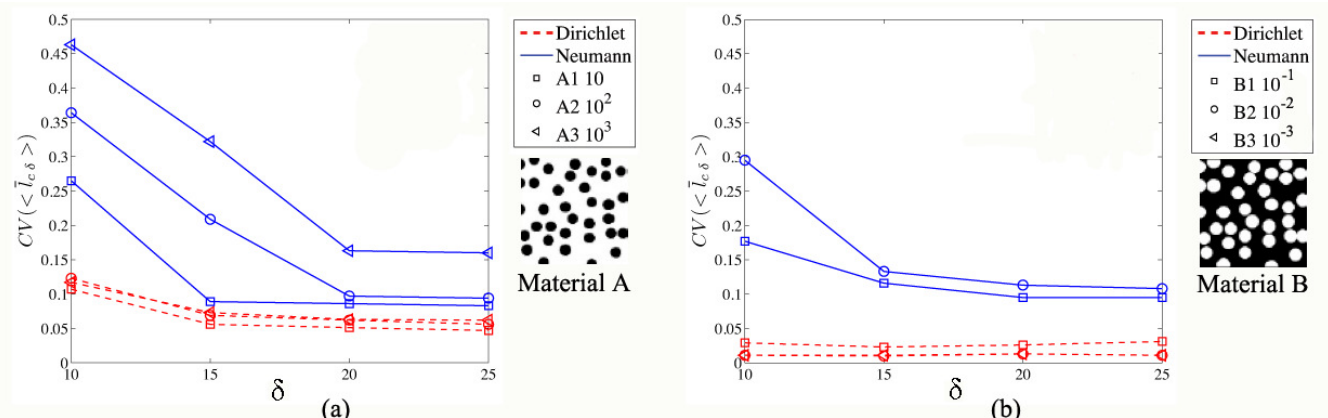

Figure 6: Material A/B: Coefficient of variation of the effective characteristic length $<\bar{l}_{c \delta}>$

Finally, the results in term of RVE size, $\delta_{R E V}$, are synthesized and reported in Figures (7) and (8). The $\delta=\delta_{R V E}$ are plotted versus the different 
contrasts considered. It can be observed that as the material contrast differs from 1 , the value of homogeneous media, the parametric analyses, obtained for different material contrasts, show that $\delta_{R V E}$ increases: the adoption of larger $\delta$ is needed in order to identify the appropriate RVE.

Accordingly, looking at figure 3 and 4 , with the adopted convergence criterion the number of simulation $N_{\delta}$ needed to reach the $\delta_{R V E}$ grows as the value of the contrast moves away from 1.

Moreover, material $\mathrm{B}$ provides more dispersed results and requires an higher number of simulations $N_{\delta}$.

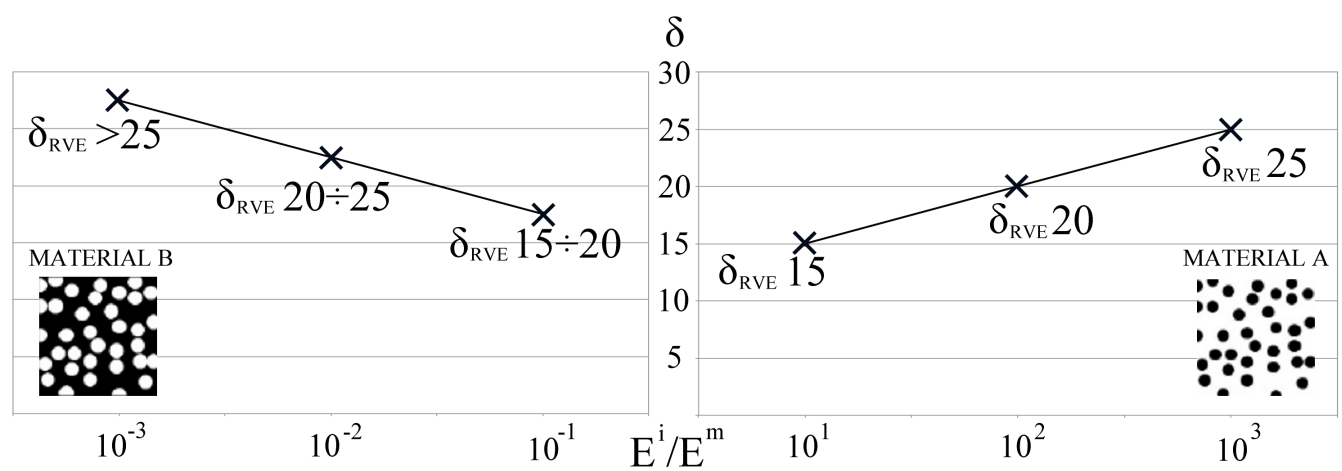

Figure 7: Material A/B: RVE size for bulk modulus for different material contrasts

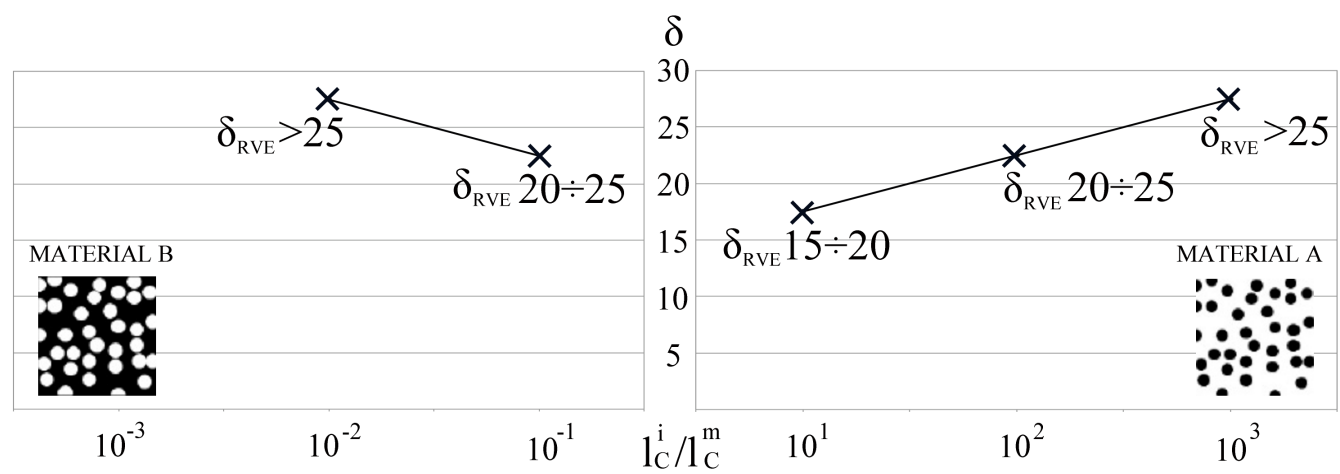

Figure 8: Material A/B: RVE size for characteristic length for different material contrasts 


\section{$5 \quad$ Final remarks}

In this work, particle composites, made of disk shaped inclusions randomly distributed within a base matrix, are investigated adopting a micropolar statistically-based homogenization procedure, previously proposed by some of the authors. The main focus is studying the sensitivity of the overall micropolar response to the mismatch between the mechanical properties of components, that is to the classical and micropolar contrasts between the elastic properties of matrix and inclusions. To this end, a set of parametric analyses have been performed for several material contrasts. Two different material cases have been considered: one with stiff inclusions (referred in the paper as material "A") and the other with soft inclusions (referred in the paper as material "B"). Particular attention is focused on the statistical convergence criterion proposed in order to evaluate the applicability and reliability of the homogenization approach at varying of the material contrast.

The outcome of the parametric analyses is the identification of the RVE (representative volume element) for a given material contrast.

Some final remarks may be drawn:

- The presented results show that the adopted scale-dependent multiscale procedure allows us to properly estimate the classical and micropolar constitutive moduli of particle-based composites described as micropolar continua.

- The window size needed to define the appropriate RVE increases as the material contrast differ from 1 (corresponding to homogeneous media).

- In both material cases (stiff or soft inclusions) randomness requires that the window intersect the inclusions.

- At varying of the contrast, the convergence trend changes depending on the constitutive moduli considered: the convergence is reached when the number of simulation $N_{\delta}$ needed to obtain average values of the constitutive parameters falling in a confidence interval is enough small. The RVE size $\delta_{R V E}$ is defined considering the most unfavorable case between the solution of Dirichlet and Neumann BVPs.

- In the case of material A (stiff inclusions) Neumann BCs provide a smaller $\delta_{R V E}$ with respect to Dirichlet BCs, while the opposite occurs in the case of material B (soft inclusions).

- The convergence criterion adopted allows us determining the appropriate RVE size to be used for performing homogenization also at sensitive varying of the contrast. 
Next step of this research is to investigate several contrasts in order to provide more significant indications to the RVE size that has to be adopted for different random composite materials. Whit this purpose, an automation of the procedure is under definition, as the computational efforts related to the statistical procedure adopted is very expensive. Moreover, the number of simulation needed to reach the convergence for materials with contrast far away from 1 is very high, calling for greater RVE size $L_{R V E}$, in particular in the case of materials with soft inclusions (B). In these cases, the mesh refinement needed using classical FE makes the computational efforts even more expensive. In this view, more efficient numerical methods will be adopted in order to adopt more coarse mesh allowing the analysis of larger SVEs.

\section{6 acknowledgments}

This work is supported by Italian Ministry of University and Research (P.R.I.N.2015, Sapienza Research Unit) and bySapienza University Grant 2016. The authors wish to thank Dr. Stefano Marcelli (Department of Market and Payment System Oversight, Bank of Italy) for the methodological support in developing the statistical procedures and for the implementation of the relating software.

\section{References}

[1] D. Addessi, M.L. De Bellis, and E. Sacco. Micromechanical analysis of heterogeneous materials subjected to overall cosserat strains. Mechanics Research Communications, 54:27 - 34, 2013.

[2] A. Bacigalupo and L. Gambarotta. Computational dynamic homogenization for the analysis of dispersive waves in layered rock masses with periodic fractures. Computers and Geotechnics, 56:61 - 68, 2014.

[3] D. Baraldi, E. Reccia, and A. Cecchi. In plane loaded masonry walls: Dem and fem/dem models. a critical review. Meccanica, Jun 2017.

[4] R. Barretta, L. Feo, R. Luciano, and F. Marotti de Sciarra. An eringenlike model for timoshenko nanobeams. Composite Structures, 139:104 110, 2016.

[5] L. Bouaoune, Y. Brunet, A. El Moumen, T. Kanit, and H. Mazouz. Random versus periodic microstructures for elasticity of fibers reinforced composites. Composites Part B: Engineering, 103:68 - 73, 2016. 
[6] F. Bouyge, I. Jasiuk, and M. Ostoja-Starzewski. Micromechanically based couple-stress model of an elastic two-phase composite. International Journal of Solids and Structures, 38(10-13):1721-1735, 2001.

[7] A. Cecchi and K. Sab. A multi-parameter homogeneization study for modelling elastic masonry. European Journal of Mechanics A/Solids, 21:249-268, 2002.

[8] A. Cecchi and K. Sab. Discrete and continuous models for in plane loaded random elastic brickwork. European Journal of Mechanics, A/Solids, 28(3):610-625, 2009.

[9] P. A. Cundall. Formulation of a three-dimensional distinct element model-part i. a scheme to detect and represent contacts in a system composed of many polyhedral blocks. International Journal of Rock Mechanics and Mining Sciences and, 25(3):107-116, 1988.

[10] P. A. Cundall and O. D. L. Strack. Discrete numerical model for granular assemblies. Geotechnique, 29(1):47-65, 1979.

[11] M. L. De Bellis and D. Addessi. A Cosserat based multi-scale model for masonry structures. International Journal for Multiscale Computational Engineering, 9(5):543-563, 2011.

[12] Y. Djebara, A. El Moumen, T. Kanit, S. Madani, and A. Imad. Modeling of the effect of particles size, particles distribution and particles number on mechanical properties of polymer-clay nano-composites: Numerical homogenization versus experimental results. Composites Part B: Engineering, 86:135 - 142, 2016.

[13] N. Fantuzzi, L. Leonetti, P. Trovalusci, and F. Tornabene. Some novel numerical applications of cosserat continua. submitted to International Journal of Computational Methods, 2017.

[14] L. Feo, F. Fraternali, and R. E. Skelton. Special issue on composite lattices and multiscale innovative materials and structures. Composites Part B: Engineering, 115:1 - 2, 2017. Composite lattices and multiscale innovative materials and structures.

[15] S. Forest and K. Sab. Cosserat overall modeling of heterogeneous materials. Mechanics Research Communications, 25(4):449 - 454, 1998.

[16] I. M. Gitman, H. Askes, and L. J. Sluys. Representative volume: Existence and size determination. Engineering Fracture Mechanics, 74(16):2518-2534, 2007. 
[17] F. Greco, L. Leonetti, R. Luciano, and P. Trovalusci. Multiscale failure analysis of periodic masonry structures with traditional and fiberreinforced mortar joints. Composites Part B: Engineering, 118:75 - 95, 2017.

[18] H. Haddad, W. Leclerc, M. Guessasma, C. Pelegris, N. Ferguen, and E. Bellenger. Application of dem to predict the elastic behavior of particulate composite materials. Granular Matter, 17:459-473, 2015.

[19] R. Hill. Elastic properties of reinforced solids: Some theoretical principles. Journal of the Mechanics and Physics of Solids, 11(5):357 - 372, 1963.

[20] Y. Ismail, D. Yang, and Y. Ye. Discrete element method for generating random fibre distributions in mechanichal models of fibre reinforced composites laminates. Composites Part B:Engineering, 90:485492, 2016.

[21] T. Kanit, S. Forest, I. Galliet, V. Mounoury, and D. Jeulin. Determination of the size of the representative volume element for random composites: statistical and numerical approach. International Journal of Solids and Structures, 40(1314):3647 - 3679, 2003.

[22] J.V. Lemos. Discrete element modeling of masonry structures. International Journal of Architectural Heritage, 1(2):190-213, 2007.

[23] P. B. Loureno, G. Milani, A. Tralli, and A. Zucchini. Analysis of masonry structures: Review of and recent trends in homogenization techniques. Canadian Journal of Civil Engineering, 34(11):1443-1457, 2007.

[24] P. R. Onck. Cosserat modeling of cellular solids. Comptes Rendus Mecanique, 330:717-722, 2002.

[25] M. Ostoja-Starzewski. Random field models of heterogeneous materials. International Journal of Solids and Structures, 35(19):2429-2455, 1998.

[26] M. Ostoja-Starzewski. Material spatial randomness: From statistical to representative volume element. Probabilistic Engineering Mechanics, 21:112-132, 2006.

[27] A. Pau and P. Trovalusci. Block masonry as equivalent micropolar continua: the role of relative rotations. Acta Mechanica, 223(7):1455-1471, 2012 . 
[28] K. Sab and B. Nedjar. Periodization of random media and representative volume element size for linear composites. Comptes Rendus - Mecanique, 333(2):187-195, 2005.

[29] T. Sadowski and L. Marsavina. Multiscale modelling of two-phase ceramic matrix composites. Computational Materials Science, 50(4):1336 $-1346,2011$.

[30] T. Sadowski and B. Pankowski. Numerical modelling of two-phase ceramic composite response under uniaxial loading. Composite Structures, 143:388 - 394, 2016.

[31] V. Sansalone, P. Trovalusci, and F. Cleri. Multiscale modelling of materials by a multifield approach: microscopic stress and strain distribution in fiber-matrix composites. Acta Materialia, 54(13):3485-3492, 2006.

[32] H. M. Shodja and B. Alemi. Effective shear modulus of solids reinforced by randomly oriented-/aligned-elliptic nanofibers in couple stress elasticity. Composites Part B: Engineering, 117:150 - 164, 2017.

[33] I. Stefanou, J. Sulem, and I. Vardoulakis. Three-dimensional Cosserat homogenization of masonry structures: elasticity. Acta Geotechnica, 3:71-83, 2008.

[34] K. Terada, M. Hori, T. Kyoya, and N. Kikuchi. Simulation of the multiscale convergence in computational homogenization approach. International Journal of Solids and Structures, 37:2285-2311, 2000.

[35] P. Trovalusci and G. Augusti. A continuum model with microstructure for materials with flaws and inclusions. Journal de Physique $I V$, Pr8:383-390, 1998.

[36] P. Trovalusci, M. L. De Bellis, and R. Masiani. A multiscale description of particle composites: From lattice microstructures to micropolar continua. Composites Part B: Engineering, 128:164 - 173, 2017.

[37] P. Trovalusci and R. Masiani. Non-linear micropolar and classical continua for anisotropic discontinuous materials. International Journal of Solids and Structures, 40:1281-1297, 2003.

[38] P. Trovalusci, M. Ostoja-Starzewski, M. L. De Bellis, and A. Murrali. Particulate random composites homogenized as micropolar materials. Meccanica, 49(9):2719-2727, 2015. 
[39] P. Trovalusci, M. Ostoja-Starzewski, M. L. De Bellis, and A. Murrali. Scale-dependent homogenization of random composites as micropolar continua. European Journal of Mechanics A/Solids, 49:396-407, 2015.

[40] P. Trovalusci and A. Pau. Derivation of microstructured continua from lattice systems via principle of virtual works. The case of masonry-like materials as micropolar, second gradient and classical continua. Acta Mechanica, 225(1):157-177, 2014.

[41] P. Trovalusci, V. Varano, and G. Rega. A generalized continuum formulation for composite materials and wave propagation in a microcracked bar. Journal of Applied Mechanics, 77(6):061002-1/11, 2010.

[42] S. Yashiro. Application of particle simulation methods to composite materials: a review. Advanced Composite Materials, 26(1):1-22, 2017.

[43] J. Zeman and M. Sejnoha. From random microstructures to representative volume elements. Modelling and Simulation in Materials Science and Engineering, 15(4):S325-S335, 2007. 